University of New Hampshire

University of New Hampshire Scholars' Repository

$11-2012$

\title{
Calibration of multibeam echo sounders: a comparison between two methodologies
}

Carlo Lanzoni

University of New Hampshire, Durham

Thomas C. Weber

University of New Hampshire, Durham, thomas.weber@unh.edu

Follow this and additional works at: https://scholars.unh.edu/ccom

Part of the Oceanography and Atmospheric Sciences and Meteorology Commons

\section{Recommended Citation}

Lanzoni, Carlo and Weber, Thomas C., "Calibration of multibeam echo sounders: a comparison between two methodologies" (2012). European Conference on Underwater Acoustics. 835.

https://scholars.unh.edu/ccom/835

This Conference Proceeding is brought to you for free and open access by the Center for Coastal and Ocean Mapping at University of New Hampshire Scholars' Repository. It has been accepted for inclusion in Center for Coastal and Ocean Mapping by an authorized administrator of University of New Hampshire Scholars' Repository. For more information, please contact Scholarly.Communication@unh.edu. 


\title{
Proceedings of Meetings on Acoustics
}

\section{ECUA 2012 11th European Conference on Underwater Acoustics \\ Edinburgh, Scotland \\ 2 - 6 July 2012}

Session UW: Underwater Acoustics

\section{UW41. Calibration of multibeam echo sounders: a comparison between two methodologies}

\author{
Jose C. Lanzoni* and Thomas C. Weber
}

*Corresponding author's address: Center for Coastal and Ocean Mapping, University of New Hampshire, 24

Colovos Rd., Durham, New Hampshire 03824, clanzoni@ ccom.unh.edu

Multibeam echo sounders (MBES) are widely used in applications like seafloor imaging, fisheries, and habitat mapping. Calibration of acoustic backscatter is an important aspect of understanding and validating the performance of a MBES. Combined transmit/receive beam pattern calibrations were performed on a $200 \mathrm{kHz}$ Reson Seabat $7125 \mathrm{MBES}$ in the acoustic tank of the University of New Hampshire utilizing two different methodologies. The first methodology employs fixed standard target spheres and a high accuracy/high resolution rotation mechanism. This method, similar to that proposed by Foote et al ["Protocols for calibrating multibeam sonar", J. Acoust. Soc. Am. 117(4), 2005], is designed for a calibration tank and provides accurate results but requires a large amount of operation time and cannot be performed in situ. The second methodology has been designed for field calibration of MBES. It employs a standard target sphere and a $200 \mathrm{kHz}$ Simrad EK60 split-beam sonar system to provide athwartship and alongship angular information of the target sphere position. This method offers the possibility of field calibration for vessel mounted systems and a significantly reduced operation time, but has a potential reduction in accuracy. In this paper, results from these two methods applied to the same MBES are compared.

Published by the Acoustical Society of America through the American Institute of Physics 


\section{INTRODUCTION}

The use of multibeam echo sounders (MBES) has grown in a variety of applications due to their high efficiency and resolution, allowing large areas to be surveyed very rapidly. Acoustic backscatter calibration increases the utility of multibeam echo sounders for several of these applications, including habitat mapping and fisheries surveys. Different procedures to calibrate sonar systems in controlled environments (e.g., tank facilities) and in the field have been developed. Conventional tank calibrations can bring excellent results in terms of resolution and accuracy, but come at the expense of large operational time and the need of the removal of the system from its operating platform. This type of calibration may not account for possible acoustic interference caused by objects close to the mounted transducers. On the other hand, a field calibration procedure can reduce the operating time and account for ship-mount interference at the expense of reduced accuracy and resolution.

Two calibration methodologies were employed to determine the combined transmit/receive radiation beam pattern of a $200 \mathrm{kHz}$ Reson Seabat 7125 MBES using the acoustic tank of Chase Ocean Engineering Laboratory at the University of New Hampshire. The tank has dimensions of $18 \mathrm{~m}$ long, 12 $\mathrm{m}$ wide, and $6 \mathrm{~m}$ deep, allowing combined transmit/receive beam pattern measurements for ranges of up to $8 \mathrm{~m}$ without interference from the sidewalls. The first methodology employs four tungsten carbide target spheres with diameters of $38.1 \mathrm{~mm}, 28.5 \mathrm{~mm}, 25 \mathrm{~mm}$, and $22.2 \mathrm{~mm}$ at ranges of $8 \mathrm{~m}, 6 \mathrm{~m}, 4 \mathrm{~m}$, and $2 \mathrm{~m}$, respectively, using a high accuracy/high resolution rotation system to position the MBES transducers, a method similar to that proposed by Foote at al [1] which is designed for tank calibration. The second methodology was designed for field calibration and employs a $200 \mathrm{kHz}$ Simrad EK60 splitbeam sonar system, operating passively, to provide the position information of a $38.1 \mathrm{~mm}$ diameter tungsten carbide target sphere while the target moves freely through the transducer beams at an approximate range of $8 \mathrm{~m}$. In each case, the target spheres were suspended in the water column by 10 Ibs. test braided monofilament lines. These lines were chosen for being very thin in order to minimize acoustic interference during the beam pattern measurements. The MBES was configured for 256 beams in equi-angle mode and set to transmit sinusoidal signals with a pulse length of $260 \mu \mathrm{s}$ for both calibration procedures. The pulse length value was chosen to maximize the performance of the splitbeam sonar system, as described in [2].

Both calibration procedures employed the same mount configuration for the transducers, although the split-beam transducer was not used for the procedure which uses the four target spheres and the rotation system to obtain high resolution calibration. The transducers of both sonar systems were installed in a rigid metallic structure as depicted by Figure 1. The split-beam transducer, a $200 \mathrm{kHz}$ Simrad ES200-7C, was placed on the $y$-axis of the multibeam system, with a separation distance of $0.955 \mathrm{~m}$ between their geometric centers. This distance was chosen to minimize possible mechanical vibration and flexing, while avoiding acoustic interference between the two sonar systems. The mounting structure was held by a carbon fiber pole, fixed at the gravity center of the mount and secured by the chucks of the high accuracy/high resolution rotation mechanism located at the top of the powered cart of the tank bridge. 
The transducers and the target spheres were positioned in the tank avoiding effects of acoustic signals reflected from the water surface, the bottom, and the sidewalls of the tank during the calibration measurements. The transducers were placed at the mid-depth of the tank $(3 \mathrm{~m})$, at $2 \mathrm{~m}$ from the back wall, and at $9 \mathrm{~m}$ from each of the sidewalls. The athwartship direction for both sonar systems is on the $y$-axis, while the alongship direction is on the z-axis. Figure 2 shows the tank setup.
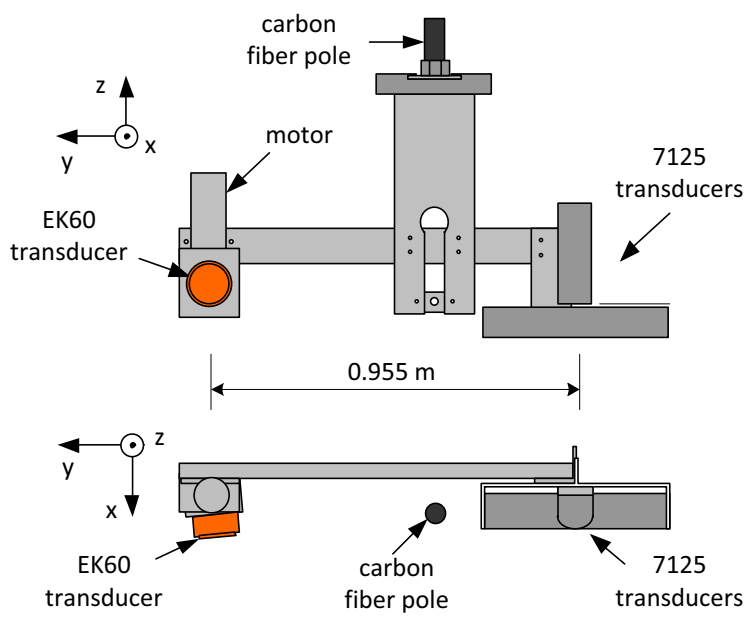

Figure 1. Transducer mount.

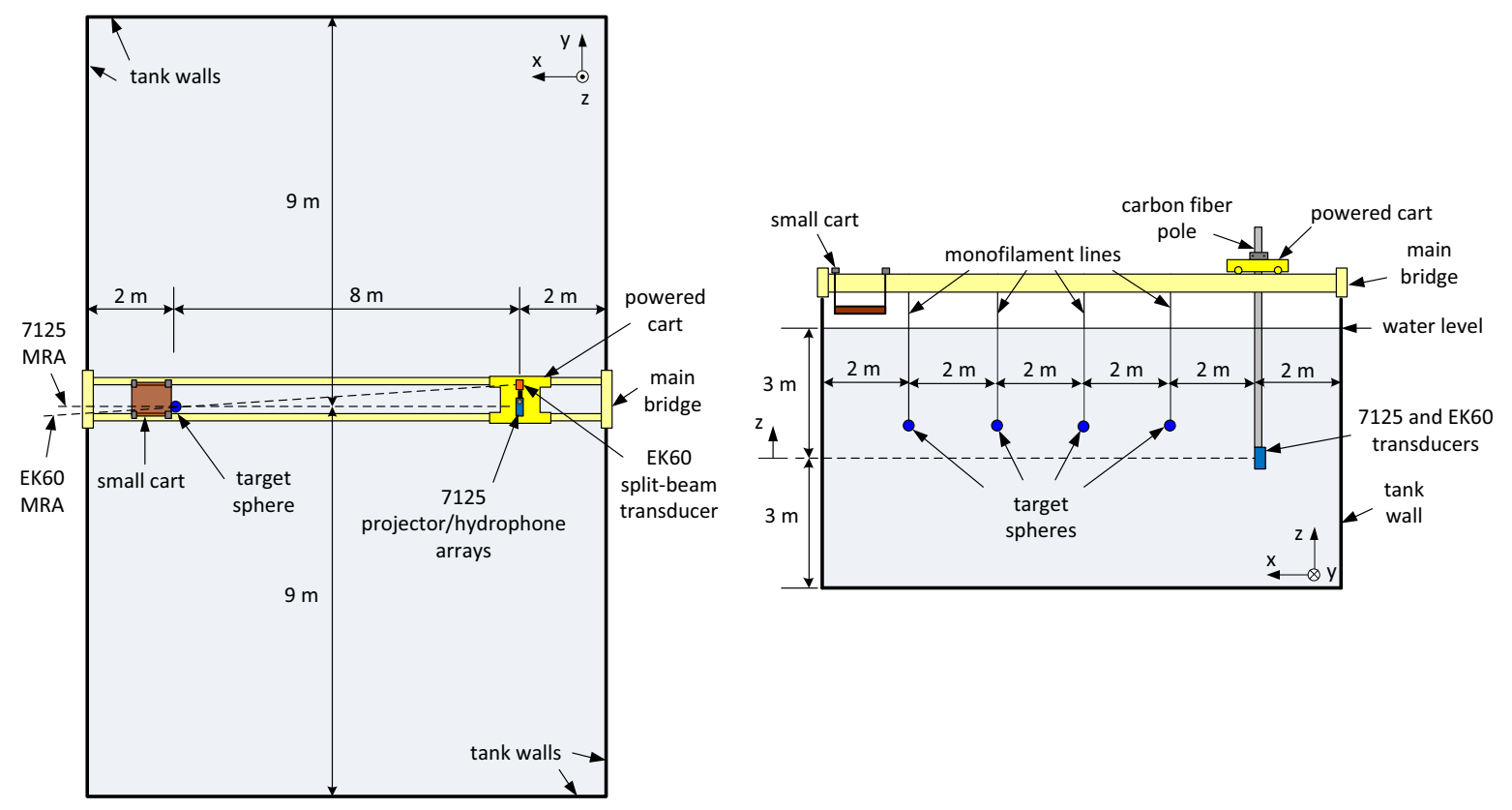

Figure 2. Setup in the acoustic tank. 


\section{CALIBRATION METHODOLOGIES OVERVIEW}

\subsection{Standard Target Spheres with Rotation Mechanism Methodology}

The first calibration methodology employed a high accuracy/high resolution rotation mechanism to control the athwartship position of the targets from the multibeam coordinates. This control was performed by a program code written in LabVIEW running on a personal computer, which also provided a trigger signal to synchronize the operation of the multibeam system, setting the desired number of pings per athwartship angular position. The MBES processing unit and the computer running LabVIEW were synchronized using the Network Time Protocol (NTP) on a point-to-point network. Beamformed data was recorded by the multibeam system, while the LabVIEW code recorded the athwartship angles and the time stamp of the pings for each athwartship position.

The four target spheres with diameters of $38.1 \mathrm{~mm}, 28.5 \mathrm{~mm}, 25 \mathrm{~mm}$, and $22.2 \mathrm{~mm}$ were placed at distances of $8 \mathrm{~m}, 6 \mathrm{~m}, 4 \mathrm{~m}$, and $2 \mathrm{~m}$, respectively, from the multibeam transducers. Each one of the target spheres was suspended in the water column by a $10 \mathrm{lbs}$. test braided monofilament line guided by a pulley system and attached to a measuring tape on the side of the tank. The tip of each measuring tape was fixed to the side of the tank and the height of the corresponding sphere was controlled by adjusting the length of the measuring tape. Figure 3 depicts the described setup to control the position of the target spheres relative to the MBES. Figure 4 shows the top view of the target spheres setup.

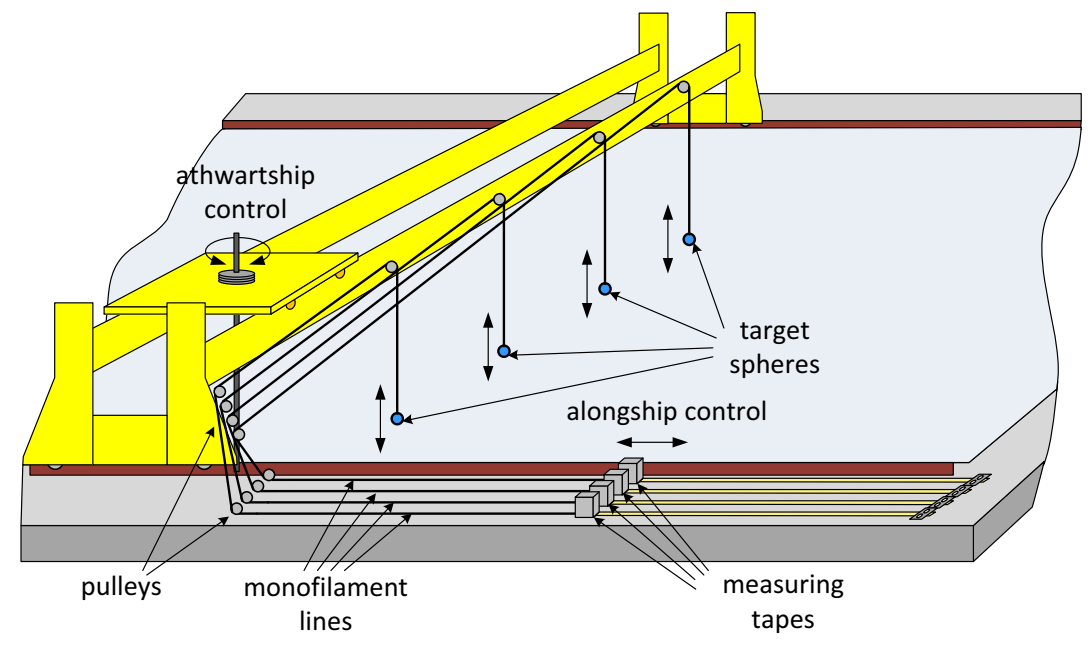

Figure 3. Positioning system for the target spheres.

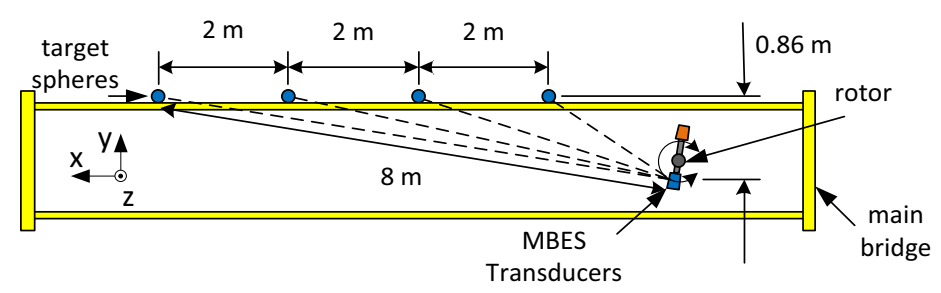

Figure 4. Target spheres setup - top view. 


\subsection{Field Calibration Methodology}

This methodology was designed for field calibration of MBES and it employs a $200 \mathrm{kHz}$ Simrad ES2007C split-beam sonar to provide the angular position of a target sphere in both alongship and athwartship directions. The target sphere, a tungsten carbide $38.1 \mathrm{~mm}$ diameter with target strength of $-39 \mathrm{~dB}$ at $200 \mathrm{kHz}$, was suspended in the water column by a $10 \mathrm{lbs}$. test braided monofilament line at a range of approximately $8 \mathrm{~m}$. The split-beam system was set to operate passively, triggered by the MBES. Beamformed data are recorded by the MBES and used to compute the target range from the MBES and the amplitude of the return signals corresponding to the target position. Data recorded by the split-beam system are used to compute the corresponding athwartship and alongship angular coordinates of the target sphere. The two systems were also synchronized using NTP on a point-topoint network, allowing for possible missing pings on the recorded data from both systems to be accounted for. Figure 5 shows the field calibration methodology overview.

The $-3 \mathrm{~dB}$ beamwidth of the split-beam system is of $7.1^{\circ}$ in both athwartship and alongship directions, which limits the angular range of the beam pattern measurements. For this reason, a motor controller was employed to adjust the split-beam transducer position in the athwartship direction. A measurement set is taken after setting the split-beam transducer to point its maximum response axis (MRA) to the center of an area of interest while moving the target sphere around this area. This process is repeated several times to encompass the whole range of beams of the MBES.

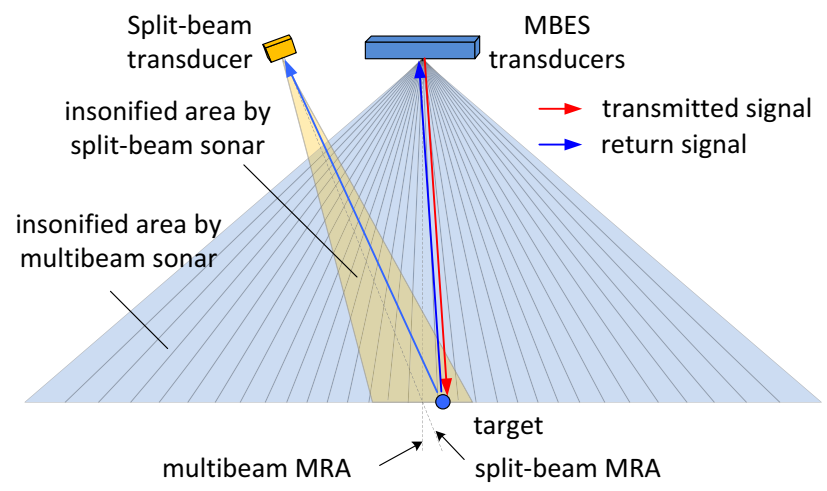

Figure 5. Field calibration methodology overview.

\section{BEAM PATTERN MEASUREMENTS}

To proper calibrate a sonar system it is necessary that it operates in linear regime during the calibration procedure, avoiding regions of non-linearities and saturation, as discussed in [3] and in more detail in [4]. For this reason, measurements were conducted to determine proper settings for transmitted power and received gain for the MBES to operate in a linear regime during the calibration tests with the four target spheres positioned at the specified ranges employing the method described in [4]. With the MBES receiver fixed gain set to the mid-range value of $40 \mathrm{~dB}$, measurements were collected for power values ranging from $170 \mathrm{~dB}$ to $220 \mathrm{~dB}$ in $1 \mathrm{~dB}$ increments. The magnitude of the signal returns corresponding to each of the target spheres recorded by the MBES were used to compute the gain curves, allowing the determination of a proper transmitted power setting at the MBES. Figure 5 shows the gain curves for targets at ranges of $2 \mathrm{~m}, 4 \mathrm{~m}, 6 \mathrm{~m}$, and $8 \mathrm{~m}$, along with a linear fit line for the curve corresponding to the $8 \mathrm{~m}$ target. 


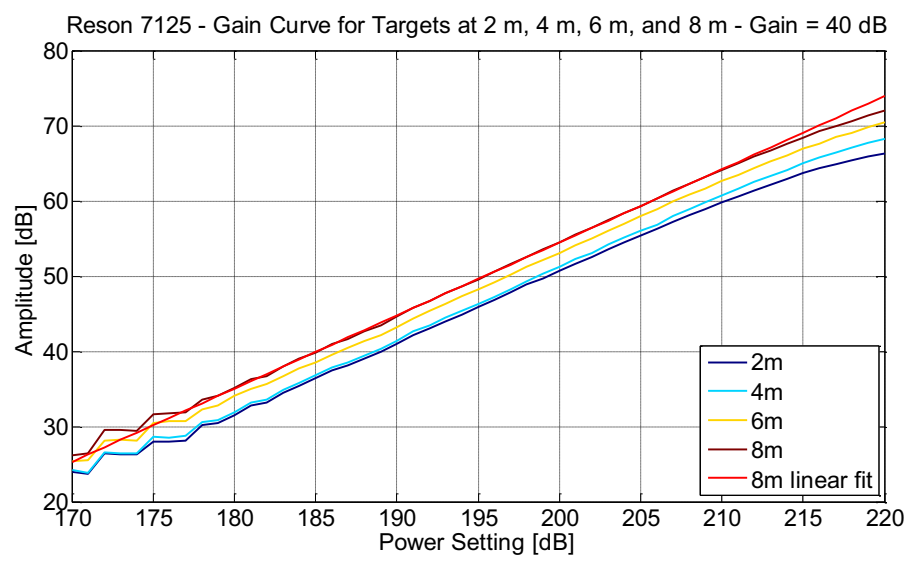

Figure 5. Reson 7125 gain curves for targets at $2 \mathrm{~m}, 4 \mathrm{~m}, 6 \mathrm{~m}$, and $8 \mathrm{~m}$.

When choosing the transmitted power setting for the MBES it is important to keep in mind that it is desirable to work with high transmitted power values during the field calibration procedure to maximize the performance of the split-beam system. According to figure 5 , the value on the curve for the $8 \mathrm{~m}$ target corresponding to the transmitted power of $215 \mathrm{~dB}$ is below the $1 \mathrm{~dB}$ compression point $(0.6 \mathrm{~dB})$, while the corresponding values for this power setting on the curves for the other targets are lower than $0.6 \mathrm{~dB}$, making these values of received gain and power setting appropriate for the two described beam pattern calibration procedures.

\subsection{Standard Target Spheres with Rotation Mechanism Measurements}

This calibration procedure was performed for an alongship range from $-12.3^{\circ}$ to $+7.5^{\circ}$ in $0.29^{\circ}$ increments for the $2 \mathrm{~m}$ target, from $-5.8^{\circ}$ to $4.2^{\circ}$ in $0.14^{\circ}$ increments for the $4 \mathrm{~m}$ target, from $-6.4^{\circ}$ to $6.8^{\circ}$ in $0.19^{\circ}$ increments for the $6 \mathrm{~m}$ target, and from $-4.9^{\circ}$ to $5.1^{\circ}$ in $0.14^{\circ}$ increments for the $8 \mathrm{~m}$ target. Alongship angles were later corrected to compensate for alongship angular offsets after computing the preliminary radiation beam patterns of the MBES corresponding to each target sphere range. The athwartship angular range was set for the rotation mechanism for the range of $+/-68^{\circ}$ in $0.2^{\circ}$ with the most inner beams of the MBES (beams 128 and 129) centered at the target sphere positioned at $6 \mathrm{~m}$. Athwartship angles were corrected for each target range to compensate for parallax errors in athwartship angular positions due to the distance from the geometric center of the MBES transducers to the center of rotation $(0.4 \mathrm{~m})$. Also, another correction in athwartship angles was performed to compensate for athwartship angular offsets.

Figure 6 shows the resulting beam pattern of the Reson 7125 MBES for beam 129 for the ranges of 2 $\mathrm{m}, 4 \mathrm{~m}, 6 \mathrm{~m}$, and $8 \mathrm{~m}$. The $-3 \mathrm{~dB}$ beamwidth in the athwartship direction for beam 129 is of approximately $1.2^{\circ}$ for the four measured ranges, while the specifications from the manufacturer state a value of $1.0^{\circ}$. In the alongship direction, the $-3 \mathrm{~dB}$ beamwidth for beam 129 is of approximately $6.2^{\circ}$ for the range of $2 \mathrm{~m}, 2.7^{\circ}$ for the range of $4 \mathrm{~m}$, and $2.4^{\circ}$ for the ranges of $6 \mathrm{~m}$ and $8 \mathrm{~m}$. Figure 7 shows the $-3 \mathrm{~dB}$ beamwidth of beam 129 for the $8 \mathrm{~m}$ range. 

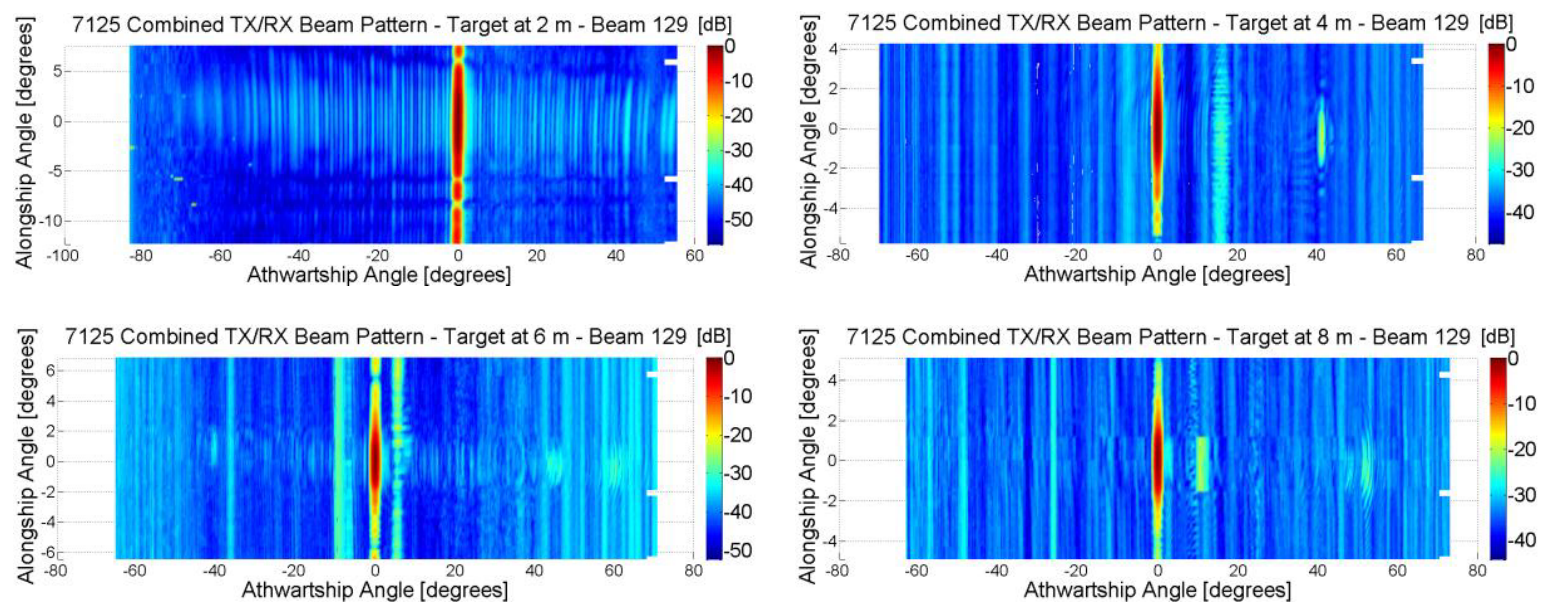

Figure 6. Beam pattern of Reson 7125 for ranges of $2 \mathrm{~m}, 4 \mathrm{~m}, 6 \mathrm{~m}$, and $8 \mathrm{~m}$ - beam 129
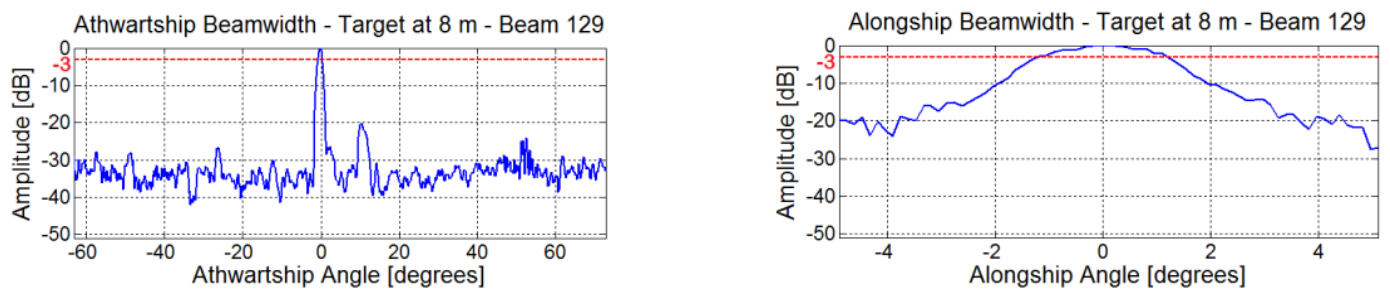

Figure 7. Athwartship and alongship -3 dB beamwidth for range of $8 \mathrm{~m}$ - beam 129 .

\subsection{Field Calibration Methodology Measurements}

This methodology requires a first measurement where the target sphere is placed at the MRA of the MBES and the split-beam transducer is positioned with its MRA pointing to the target in the athwartship direction. This is necessary to compute the time delay between the triggering of both sonar systems which is used to convert the position of the target from the split-beam coordinates to the MBES coordinates in the data processing stage. The motor controller that adjusts the split-beam transducer is set to $0^{\circ}$ at this position. A set of measurements is taken for the split-beam athwartship angular positions ranging from $-60^{\circ}$ to $+65^{\circ}$ in $5^{\circ}$ increments with the target sphere moving from a range of approximately $+/-7^{\circ}$ from the split-beam MRA for each position (athwartship and alongship directions). For each set of measurements, the rotation mechanism from the main bridge was used to adjust the position of the split-beam MRA in athwartship direction to point to a reference position which is the center of the area where the target sphere was moved. This way, the whole range of beams of the MBES was covered with the target at a range of approximate $8 \mathrm{~m}$.

An indication of possible acoustic interference caused by the monofilament line used to hold the target sphere was observed in the angular measurements from the split-beam system. This problem was minimized by setting a threshold value for the point backscattering strength (Sp) during the data processing of the split-beam system record. Figure 8 shows values of alongship and athwartship angles from the split-beam system using $\mathrm{Sp}$ threshold values of $-70 \mathrm{~dB}$ and $-60 \mathrm{~dB}$. As observed from this figure, values of athwartship and alongship angles corresponding to the target become less noisy as the $\mathrm{Sp}$ threshold value is increased at the cost of a smaller alongship angular range. The radiation 
beam pattern of the MBES was computed using the $S p$ threshold value of $-60 \mathrm{~dB}$. Figure 9 shows the computed beam pattern for beam 129.
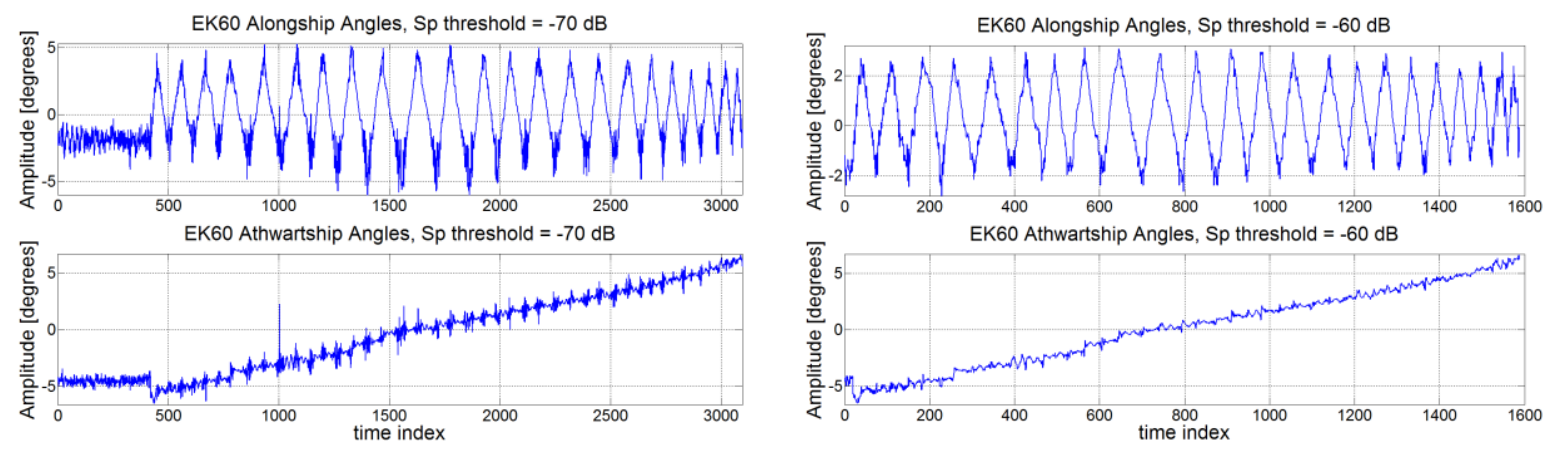

Figure 8. EK60 athwartship and alongship angles for Sp threshold of $-70 \mathrm{~dB}$ and $-60 \mathrm{~dB}$.

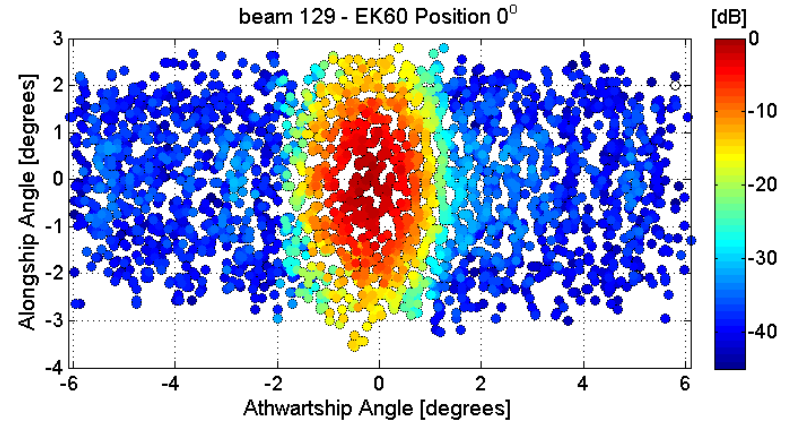

Figure 9. Reson 7125 beam pattern - beam 129.

Figure 10 shows the beamwidth in athwartship and alongship directions for beam 129. The $-3 \mathrm{~dB}$ beamwidth for this beam in the athwartship direction is of approximately $1.0^{\circ}$ and in the alongship direction it is of approximately $1.9^{\circ}$, agreeing with the values provided by the manufacturer of $1.0^{\circ}$ and $2.0^{\circ}$ respectively.
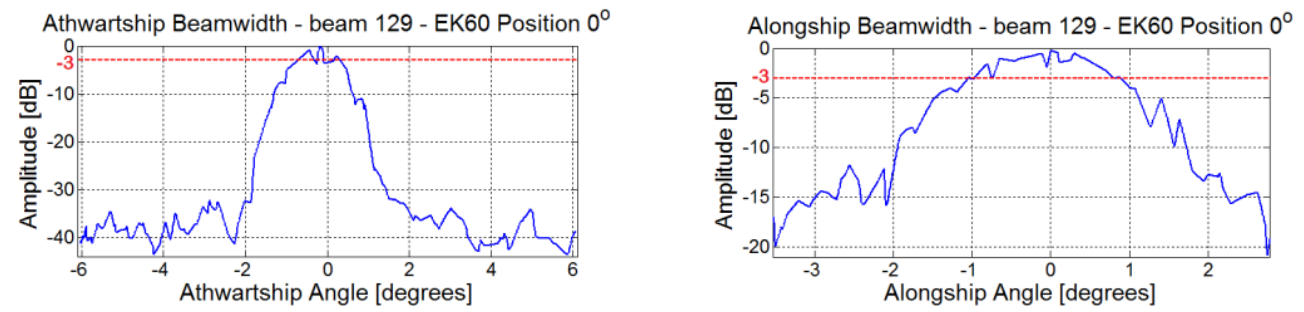

Figure 10. Athwartship and alongship $-3 \mathrm{~dB}$ beamwidth - beam 129

\section{CONCLUSION}

Combined radiation beam pattern calibrations were performed on a $200 \mathrm{kHz}$ Reson Seabat MBES in the acoustic tank at the University of New Hampshire employing two different methodologies. The first methodology employed four tungsten carbide target spheres suspended by monofilament lines and a 
high accuracy/high resolution rotation mechanism. The second methodology, designed for field calibration of MBES, employed a freely moving target sphere and a $200 \mathrm{kHz}$ Simrad EK60 split-beam echo sounder to provide the athwartship and alongship position of the target sphere during the calibration measurements. The two methodologies utilized the same configuration for the MBES: 256 beams, equi-angle mode, and transmitted signals with pulse length of $260 \mu \mathrm{s}$.

Measurements were conducted to determine proper transmitted power and receive gain settings for the MBES to work in a linear regime. These values, $220 \mathrm{~dB}$ for transmitted power and $40 \mathrm{~dB}$ for receive gain, were applied during the calibration measurements for both methodologies.

In the first employed methodology, the target spheres with diameters of $38.1 \mathrm{~mm}, 28.5 \mathrm{~mm}, 25 \mathrm{~mm}$, and $22.2 \mathrm{~mm}$ were placed at ranges of $8 \mathrm{~m}, 6 \mathrm{~m}, 4 \mathrm{~m}$, and $2 \mathrm{~m}$, respectively, from the multibeam transducers. The radiation beam pattern was computed for the alongship range from $-12.3^{\circ}$ to $+7.5^{\circ}$ in $0.29^{\circ}$ increments for the $2 \mathrm{~m}$ target, from $-5.8^{\circ}$ to $4.2^{\circ}$ in $0.14^{\circ}$ increments for the $4 \mathrm{~m}$ target, from $-6.4^{\circ}$ to $6.8^{\circ}$ in $0.19^{\circ}$ increments for the $6 \mathrm{~m}$ target, and from $-4.9^{\circ}$ to $5.1^{\circ}$ in $0.14^{\circ}$ increments for the $8 \mathrm{~m}$ target. The athwartship angular range was set for the rotation mechanism for the range of $+/-68^{\circ}$ in $0.2^{\circ}$ with the most inner beams of the MBES (beams 128 and 129) centered at the target sphere positioned at $6 \mathrm{~m}$. The $-3 \mathrm{~dB}$ beamwidth in the athwartship direction for beam 129 is of approximately $1.2^{\circ}$ for the four measured ranges, while the specifications from the manufacturer state a value of $1.0^{\circ}$. In the alongship direction, the $-3 \mathrm{~dB}$ beamwidth for beam 129 is of approximately $6.2^{\circ}$ for the range of $2 \mathrm{~m}$, $2.7^{\circ}$ for the range of $4 \mathrm{~m}$, and $2.4^{\circ}$ for the ranges of $6 \mathrm{~m}$ and $8 \mathrm{~m}$. The manufacturer specification for the $-3 \mathrm{~dB}$ beamwidth in the alongship direction is of $2.0^{\circ}$.

The second calibration methodology employed a motor controller to adjust the split-beam transducer MRA in the athwartship direction to cover the entire range of beams of the MBES during the beam pattern measurements for the range of $8 \mathrm{~m}$. After aligning the split-beam transducer MRA with the MBES MRA at the target range of $8 \mathrm{~m}$, the split-beam transducer position was set to $0^{\circ}$. The split beam transducer angle (in the athwartship direction) was set for the range from $-60^{\circ}$ to $+65^{\circ}$ in $5^{\circ}$ increments and a measurement set was recorded for each of these positions while the target sphere was swept between a range of $+/-7^{\circ}$ in the athwartship and alongship directions from the center of each of these positions. Indication of possible acoustic interference from the monofilament line was observed in the split-beam records. This problem was minimized by increasing the point backscattering strength (Sp) threshold value during the split-beam data processing stage, at the expense of a reduced alongship range for the computed beam pattern. Beam pattern results show a $-3 \mathrm{~dB}$ beamwidth of approximately $1.0^{\circ}$ for beam 129 in the athwartship direction and of approximately $1.9^{\circ}$ in the alongship direction, agreeing with the values provided by the manufacturer.

The first presented methodology has the advantage of providing more accurate results and the possibility of taking high resolution beam pattern measurements for different ranges at the same time. However, this method requires the removal of the system from its original operating platform, a long operational time (about two weeks), and does not account for possible acoustic interference in shipmounted systems.

The second calibration methodology presented here offers the possibility of field calibration for vessel mounted systems with significant reduced operational time when compared to conventional tank calibrations, with a potential reduction in accuracy. The effects of possible acoustic interference from the monofilament line observed from the tests in the acoustic tank may be reduced during a field calibration, where the geometry of the lines holding the target sphere would be different. 


\section{ACKNOWLEDGEMENTS}

This work was supported under NOAA Grant NA05N0S4001153. The following are thanked for their participation in the calibration experiments: P. Lavoie, V. Schmidt, University of New Hampshire, G. Rice, B. Welton, National Oceanic and Atmospheric Administration. The National Oceanic and Atmospheric Administration (NOAA) is thanked for lending the multibeam system for use in the calibration experiments.

\section{REFERENCES}

1. K.G. Foote, D. Chu, T.R. Hammar, K.C. Baldwin, L.A. Mayer, L.C. Hufnagle, Jr., , and J. M. Jech, "protocols for calibrating multibeam Sonar," J. Acoust. Soc. Am. 117(4), 2005.

2. J.C. Lanzoni and T.C. Weber, "A Method for Field Calibration of a Multibeam Echo Sounder", Proc. MTS/IEEE Oceans 2011 Conf., Sep. 19-22, Kona, HI.

3. J.C. Lanzoni and T.C. Weber, "High-resolution Calibration of a Multibeam Echo Sounder", Proc. MTS/IEEE Oceans 2010 Conf., Sep. 20-23, Seattle, WA.

4. S.F. Greenaway and T.C. Weber, "Test Methodology for Evaluation of Linearity of Multibeam Echosounder Backscatter Performance", Proc. MTS/IEEE Oceans 2010 Conf., Sep. 20-23, Seattle, WA.

5. Foote K, Knudsen H, Vestnes G, MacLennan D, Simmonds E (1987), Calibration of Acoustic instruments for fish density estimation, ICES Coop. Res. Rep. 144, $81 \mathrm{p}$.

6. K.G. Foote, D. Chu, T.R. Hammar, K.C. Baldwin, L.A. Mayer, L.C. Hufnagle, Jr., and J.M. Jech, "Protocols for Calibrating Multibeam Sonar," J. Acoust. Soc. Am., vol. 114, pp. 2013-2027, 2005.

7. W.S. Burdic, "Underwater Acoustic System Analysis," $2^{\text {nd }}$ ed., Prentice Hall, New Jersey, 1991.

8. Meinberg, Network Time Protocol (NTP), [Online], Available: http://www.meinberg.de/english/info/ntp.htm.

9. T.K. Stanton and D. Chu, "Calibration of Broadband Active Acoustic Systems Using a Single Spherical Target," J. Acoust. Soc. Am. 124, 128-136, 2008.

10. D. Chu, K.G. Foote, L.C. Hufnagle, Jr., T.R. Hammar, S.P. Liberatore, K.C. Baldwin, L.A. Mayer, and A. McLeod, "Calibrating a 90-kHz Multibeam Sonar," Proc. MTS/IEEE Oceans 2003 Conf., Sep. 22-26, San Diego, CA, pp. 1633-1636.

11. T. R. Hammar, K. G. Foote, and S. P. Liberatore, "Advances in developing a high-frequency sonar calibration facility," Proc. MTS/IEEE Oceans 2003 Conf., pp. 1622-1624.

12. K. C. Baldwin, L. Mayer, A. McLeod, and J. Millar, "Acoustic transducer calibration system," Proc. MTS/IEEE Oceans 2003 Conf., pp. 2093-2099.

13. K.G. Foote, "Maintaining Precision Calibrations with Optimal Copper Spheres," J. Acoust. Soc. Am. 73, 1054-1063 (1983).

14. D. Chu, K.G. Foote, and L.C. Jr. Hufnagle, "Measurement of Multibeam Sonar Directivity Patterns," Proc. MTS/IEEE Oceans 2002 Conf., Oct. 29-31, vol. 3, pp. 1411-1414.

15. G.C. Gaunaurd, "Sonar Cross Section of Bodies Partially Insonified by Finite Sound Beams," IEEE J. Ocean. Eng. OE-10, 213-230, 1985.

16. K.G. Foote and D.N. MacLennan, "Comparison of Copper and Tungsten Carbide Calibration Spheres," J. Acoust. Soc. Am. 75, 612-616, 1984. 